\title{
Activated platelets in patients suffering from inflammatory bowel disease
}

\author{
Tekelioglu $\mathrm{Y}^{1}$, Uzun $\mathrm{H}^{2}$, Sisman $\mathrm{G}^{3}$ \\ Karadeniz Technical University, Medical Faculty, Histology and Embryology Division, Trabzon, Turkey. \\ ytekeli61@yahoo.com
}

\begin{abstract}
Background: There is an increased risk of thromboembolic complications in inflammatory bowel disease. Activated platelets play a crucial role in the pathogenesis of this disease.

Aim: To evaluate platelet activation in inflammatory bowel disease.

Material and method: This study comprised 20 healthy control subjects and a total of 20 patients. Out of them, 4 patients and 16 patients had suffered from Crohn's disease and ulcerative colitis, respectively. Nine patients were in active phase and 11 were in inactive phase of the disease. To evaluate platelet activation, we used the monoclonal antibodies of mouse anti-human CD42a-Fluorescein isothiocyanate (FITC), CD42b-FITC and mouse anti-human CD62P-phycoerythrin. We assessed the activation of platelets in peripheral blood using flow cytometric analysis. Result: The platelet activation was found to be statistically significantly higher in the active-phase patient group when compared with the control subjects group. On the other hand, it was insignificant in the inactive patient group. Conclusion: The results of our study might suggest that the elevation of CD62P expression in patients with inflammatory bowel disease could be used as a criterion of disease activation. Furthermore, agents with properties to diminish the platelet activation could prevent the development of thromboembolic complications in a patient with inflamatory bowel disease (Fig. 1, Ref. 15). Text in PDF www.elis.sk.

Key words: activated platelets, inflammatory bowel disease, thromboembolic complications, ulcerative colitis, Crohn's disease, monoclonal antibodies.
\end{abstract}

In inflammatory bowel diseases (IBD), Crohn's disease and ulcerative colitis, the exacerbation is accompanied by thrombocytosis. For these patients, there is an increased risk of thromboembolic complications (1). In the pathogenesis of Crohn's disease, multifocal microinfarcts were detected on mesenteric veins as an early pathological finding (2).

Platelets play a crucial role in the hemostatis and inflammatory response. Selectin, which regulates the hemostasis and inflammatory response, secretes many inflammatory mediators such as platelet factor-4, CD40L, $\beta$-thromboglobuline $(1,3-5)$.

The adhesion of circulating cells to the endothelium is a multistep process. In each step, the specific families of adhesion molecules, which are expressed in the endothelial cells and circulating cells, play a role. Among these adhesion molecules, the selectin family has a role in the first step to cell adhesion. The expression of selectins increases in various tissues including the intestinal tissues of patients with $\operatorname{IBD}(1,6)$.

$\mathrm{P}$-selectin (CD62P), which is a member of selectin family, is a $140 \mathrm{kDa}$ membrane glycoprotein located in the alpha granules and dense granules of platelets and endothelial cells. Activation

${ }^{1}$ Karadeniz Technical University, Medical Faculty, Histology and Embryology Division, Trabzon, Turkey, ${ }^{2}$ Memorial Hospital, Specialist of Family Medicine, Istanbul, Turkey, and ${ }^{3}$ Istanbul University Cerrahpasa, Medical Faculty, Gastroenterology Division, Turkey

Address for correspondence: Y. Tekelioglu, Karadeniz Technical University, Medical Faculty, Histology and Embryology Division, Trabzon, Turkey. Phone: +05324423412 of these cells results in rapid mobilization of CD62P from storage granules to cellular surface. The main effect of P-selectin is to facilitate the diapedesis and adhesion of neutrophils (7).

CD42a, which is also known as platelet glycoprotein IX (GP9), is a small membrane glycoprotein found on the surface of human platelets. It forms a noncovalent complex with glycoprotein Ib (GP Ib), a platelet surface membrane glycoprotein complex that functions as a receptor for von Willebrand factor. CD42b is the alpha subunit of glycoprotein Ib. The binding of GP Ib-IX-V complex to von Willebrand factor facilitates initial platelet adhesion to vascular subendothelium after vascular injury, and also initiates signaling events within the platelet that lead to enhanced platelet activation, thrombosis, and hemostasis.

Considering the platelet activation in IBD, our aim was to measure the platelet activation in peripheral blood in patients with IBD using flow cytometric analysis and to compare the results with those of the control group in this study (Fig. 1).

\section{Material and method}

Twenty patients who had been followed up in Istanbul Private Memorial Service Hospital were studied. Among them, 4 patients had Crohn's disease while the remaining 16 patients had ulcerative colitis. Their ages ranged between 28 and 65 years and their mean age was 41.5 years. Nine patients were in active phase, while 11 patients were in inactive phase. The ulcerative colitis patients, whose score was $\geq 5$ according to the clinical activity index defined 

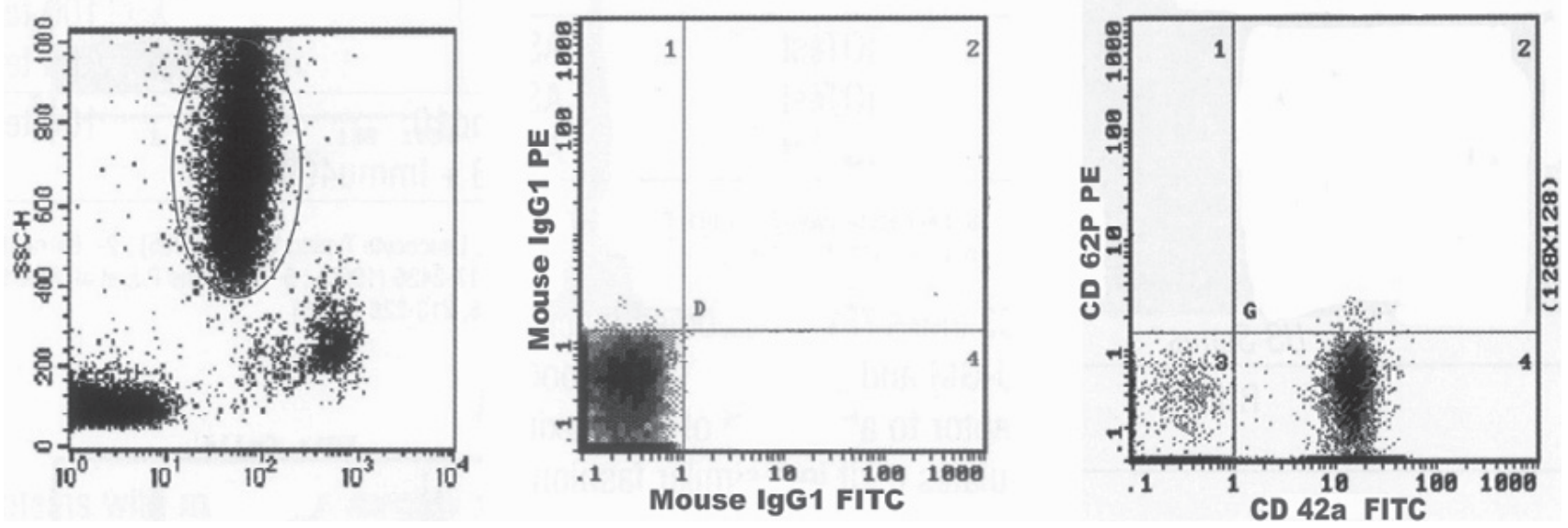

Fig. 1. Flow cytometric analysis for CD42a and CD62P expressions; first diagram shows gated cells, second diagram shows no positive data on either $x$ or $y$ axis, the third diagram shows positive data for CD42aFITC on $x$ axis (area number 4), no positive data for CD62P PE on $y$ axis (area number 1).

by Rachmilewitz (8), were regarded as active-phase patients. The Crohn's disease patients, whose score was $\geq 150$ according to the activity index defined by Best, were regarded as active-phase patients (9). All active-phase patients had ulcerative colitis. Twenty voluntary healthy subjects matched by age and sex served as a control group. Patients having arterial disease, peripheral vascular disease, hypertension, chronic obstructive pulmonary disease, diabetes mellitus, renal insufficiency, hypercholesterolemia, smoking history, and those using oral contraceptive agents and aspirin were excluded. This study was approved with the permit of the Istanbul Private Memorial Hospital. The participants signed the written informed consent.

\section{Blood sampling}

Blood samples were drawn from a vein in region without stasis, and then passed into siliconised vacutainer tubes which contained $3.6 \mathrm{mg}$ of sterilized Becton Dickinson K2E.

\section{Monoclonal antibodies}

To evaluate the platelet activation, we used monoclonal antibodies such as mouse anti-human CD42a-Fluorescein isothiocyanate (FITC) (Beckman Coulter IMI757), CD42b-FITC (Beckman Coulter IM0648) and mouse anti-human CD62P-phycoerythrin (PE) (Beckman Coulter IMI759).

\section{Flow cytometric analysis}

We used a Beckman Coulter EPICS ELITE analyser for flow cytometric analysis. Each tube (CD42a /CD62P, CD42b /CD62P) had been separately prepared for blood samples of patients and healthy subjects and added with 20 microliters of monoclonal antibody and then mixed with 20 microliters of blood sample and finally, incubated for 15 minutes at room temperature. Then, these tubes were processed by Coulter Multi Q preparation device. Immunoprep A, B, and C solutions (ImmunoprepA: formic acidstabilizer, ImmunoprepB: Sodium carbonate-chloride-sulphatestabilizer, and ImmunoprepC: Paraformaldhyde-buffers Coulter
Immunoprep reagent system PN7507950-D) were added. After 15 minutes, the samples became ready for flow cytometric analysis.

We used Forward Scatter (FSC) and Side Scatter (SSC) logarithmic histogram modes. The area which contained platelets on a dot plot of FSC and SSC histogram, was gated (R1 gate). The activation of platelets in the marked area that existed on the dot plot of CD42a/CD62P, CD42b/CD62P was analyzed.

\section{Statistical analysis}

To evaluate the data, we used Mann-Whitney U test. $\mathrm{p}<0.05$ was taken as statistically significant.

\section{Results}

The CD62P (P-selectin) expression of the patients with IBD was significantly higher when compared with the control group (The median value was 3.73 for the patient group (3.50-10.90), while the median value of the control group was found to be 1.25 ; $\mathrm{p}<0.05,(0.90-1.9))$.

The P-selectin expression in 9 patients with active IBD was found to be significantly higher when compared with the control group (The median value was $7.3(5.10-12.90)$ in the active-phase patient group, while the median value of the control group was found to be $1.62(1.1-2.1) ; \mathrm{p}<0.05)$. Although the Pselectin expression of 11 patients with inactive IBD tended to be higher, there was no statistical significance when compared with the control group. (The median value was $2.75(2.2-3.1)$ in the inactive-phase patient group, and $1.62(1.1-2.1)$ in the control group; $\mathrm{p}=0.07$ ).

When the groups of active and inactive-phase patients were compared with each other, the P-selectin expression of those in the active-phase patient group was found to be higher than that of those in the inactive-phase patient group, and statistically significant (The mean percentage value was $7.12 \pm$ SEM 1.56 for the active-phase patients, and $2.88 \pm$ SEM 0.92 for the inactive-phase patients; $\mathrm{p}<0.05)$. 


\section{Discussion}

In this study, we found that the platelet activation was significantly higher in the patient group compared to the control group.

It is thought that the mechanisms considered being the reason for platelet activation in IBD starts when the endothelial damage occurs due to chronic inflammatory process or effect of endotoxin. As a result of endothelial damage, the platelets become active after encountering the subendothelial collagen. Furthermore, the secretion of tissue factor from damaged endothelium and also the production of thrombin play a role in platelet activation. Platelet-activating factor, which is secreted from the inflamed bowel tissue, also contributes to the activation $(10,11)$. Not only play the platelets a part in hemostasis and thrombosis, they also carry proinflammatory characteristics since they secrete mediators such as platelet factor 4, platelet-activating factor, interleukin-8 and arachidonic acid metabolites. It was shown that production of all these mediators was increased in IBD (12). In addition to the physiological repair mechanisms, platelet-derived growth factor, which is secreted from platelets, also contributes to stricture formation in the inflamed area (13).

Schürmann et al assessed the surgical bowel resection materials of 58 patients with ulcerative colitis and Crohn's disease. They evaluated the endothelial platelet activation as an immunohistochemical factor and proposed that when compared with the normal bowel segment, the veins, venules and capillaries in the inflamed area showed more of P-selectin expression raised up to 7 or 8 folds. This expression showed a parallelism with the activation level of disease (6). Collins et al also indicated that expression of CD62P (P-selectin) and GP53 (surface markers of platelet activation) was increased in patients with Crohn's disease and ulcerative colitis (1). Similarly, in our study, P-selectin expression is increased in the platelet surfaces of the patients with IBD.

The study conducted by Fägerstam et al also showed that Pselectin expression was increased on platelet surfaces in patients with IBD in remission period (14). Also, Dong et al determined that the plasma soluble P-selectin level displays a parallelism with the severity of ulcerative colitis (15). In our study, when compared with the control and inactive patients group, we found that CD62P (P-selectin) expression level and accordingly the platelet activation were higher in the active-phase patient group. On the other hand, the mean CD62P level was not significantly higher in inactive patients when compared with normal controls. This situation may lead us to a conviction that elevated levels of CD62P expression in patients with IBD can serve as a criterion for active disease. Furthermore, drugs inhibiting platelet activation and thus preventing the occurrence of thromboembolic complications in the vessels of inflamed bowels could be of use in the treatment of IBD.

\section{References}

1. Collins CE, Cahili MR, Newland AC, Rampton DS. Platelets circulate in an activated state in inflammatory bowel disease. Gastroenterology 1994; 106; 840-845.

2. Wakefield AJ, Savvyerr AM, Dhillon AP, Pittiio RM, Rowles PM, Levvis AAM, Pounder RE. Pathogenesis of Crohn's disease: multifocal gastrointestinal infarction. Lancet 1989; 2: 1057-1062.

3. Danese S, de la Motte C, Sturm A, Vogal JD, West GA, Strong SA et al. Platelets trigger a CD40-dependent inflammatory response in the microvasculature of inflammatory bowel disease patients. Gastroenterology 2003; 124: 1249-1264.

4. Danese S, Katz JA, Saibeni S, Papa A, Gasbarrini A, Vecchi M et al. Activated platelets are the source of elevated levels of soluble CD40 ligand in the circulation of inflammatory bowel disease patients. Gut 2003; 52: 1435-1441.

5. Mannaioni PF, Di Bello MG, Masini E. Platelets and infl ammation: role of platelet-derived growth factor, adhesion molecules and histamine. Inflamm Res 1997; 46: 4-18.

6. Schürmann GM, Bishop AE, Facer P, Vecchio M, Lee JC, Rampton DS, Polak JM. Increased expression of celi adhesion molecule P-seiectin in active inflammatory bowel disease. Gut 1995; 36 (3): 411-418.

7. de Bruijne-Admiraal LG, Modderman PW, Von dem Borne AEGK, Sonnenberg A. P-selectin mediates $\mathrm{Ca}^{2+}$-dependent adhesion of activated platelets to many different types of leukocytes: detection by flow cytometry. Blood 1992; 80: 134-142.

8. Rachmilewitz D. Coated mesalazine (5-aminosalicylic acid) versus sulphasalazine in the treatment of active ulcerative colitis: a randomised trial. BMJ 1989; 298: 82-86.

9. Best WR, Becktel JM, Singleton JW, Kern F Jr. Development of a Crohn's disease activity index. National Cooperative Crohn's Disease Study. Gastroenterology 1976; 70: 439-444.

10. Colucci M, Balconi G, Lorenzet R, Pietra A, Locati PD, Donati MB, Semeraro N. Cultured human endothelial cells generate tissue factor in response to endotoxin. J Clin Invest 1983; 71: 1893-1896.

11. Wellmann W, Fink P, Benner F, et al. Endotoxemia in active Crohn's disease. Treatment with whole gut irrigation and 5-amino salicylic acid. Gut 1986; 27: 814-820.

12. Rainger GE, Buckley C, Simmons DL. Neutrophils rolling on immobilised platelets migrate into homotypic aggregates after activation. Thromb Haemost 1998; 79 (6):1177-1183.

13. Simmonds NJ, Ailen RE, Stevens TR, Van Someren RN, Blake DR, Rampton DS. Chemiluminescence assay of mucosal reactive oxygen metabolites in inflammatory bovvel disease. Gastroenterology 1992; 103: $186-196$

14. Fagerstam JP, Whiss PA, Strom M, Andersson RG. Expression of platelet P-selectin and detection of soluble P-selectin, NPY and RANTES in patients with inflammatory bowel disease. Inflamm Res 2000; 49: 466-472.

15. Dong WG, Liu SP, Zhu HH, Luo HS, Yu JP. Abnormal function of platelets and role of angelica sinensis in patients with ulcerative colitis. World J Gastroenterol 2004; 10: 606-609. 